Materials and Methods We defined four performance indicators and analysed data from 2011 using the Web Reporting software supplied with ADS, and compared them with the 2008 results. Data were collected from the five EP-linked units.

1. Non-prescribed dispensing (NPD): percentage of drugs dispensed with no prescription assigned. It reflects physicians' prescription mistakes, ward dispensing mistakes, or technical problems with EP and EP-ADS interface.

2. Assigned Patient (AP): NPD with assigned patient. This indicator informs us about proper use, mainly in non EPlinked ADS units.

3. Fictional Patient (FP): NPD assigned to the fictional patient every unit has. This indicator reports us about technical problems with the hospital patient census and with the EP. It can also inform us of misuse of the ADS

4. Discrepancies (DR): stock discrepancies as a percentage of global ADS transactions. These are related to ward dispensing mistakes or pharmacy supply mistakes.

Results NPD: $12.4 \%$ (25,820/208,957 drugs dispensed), lower than the 2008 results by 2.1 percentage points.

AP: $7.8 \%, 2.3$ percentage point reduction.

FP: $4.6 \%, 0.3$ percentage point increase.

DR: $3.0 \%(6,250 / 259,791$ transactions), 0.3 percentage point reduction.

Conclusions ADS performance indicators have shown effectiveness in monitoring the processes. Between 2008 and 2011 we have improved in NPD, AP and DR results, but we have to work with factors that increased FP. We have found differences between some $\mathrm{ADS}$ units so a need for additional training in some wards has been revealed.

No conflict of interest.

\section{TCH-004 CENTRALIZATION AND TECHNOLOGY SUPPORT THE HOSPITAL PHARMACIST IN IMPROVING SAFETY, ACCURACY AND ECONOMY IN THE MANAGEMENT OF MONOCLONAL ANTIBODIES}

doi:10.1136/ejhpharm-2013-000276.195

'S Corridoni, 'E Liberatore, 'AM lacomini, ' $\mathrm{G}$ Tinari, ' ${ }^{1} \mathrm{~S}$ Massacese, ${ }^{2} \mathrm{~L}$ Losavio. ${ }^{1}$ Hospital San Salvatore L'Aquila, Pharmacy, L'Aquila, Italy; ${ }^{2}$ Loccioni Group, Humancare, Moie di Maiolati Ancona, Italy

Background Antineoplastic drugs are considered 'high-risk drugs' due to the increased frequency of human technical errors in their preparation. It is essential for pharmacists to be responsible for setting up, centralising and managing cytotoxic drugs (CDs). To this end, the Division of Anticancer Drugs of L'Aquila (Italy) acquired on June 2012 a Robotic System, APOTECAchemo, the first worldwide system for chemotherapy compounding in a controlled atmosphere.

Purpose To analyse the impact of centralising and automating CD preparation for all the Departments in the Hospital of L'Aquila, to avoid any possibility of human error and to optimise the use of the remainder of CDs.

Materials and Methods Three high cost monoclonal antibodies (bevacizumab, cetuximab and trastuzumab) were chosen for analysis in this study during the period June-September 2012. The criteria for product suitability were evaluated by analysing the APOTECAchemo database in which all stages of the production process are recorded (picture of the bottle used, weight, and dose accuracy). The cost analysis was evaluated by calculating the daily amounts left over of the three drugs that were previously discarded and are now fully re-used.

Results The average error was for 168 preparations of bevacizumab $+0.45 \%$ (DS $=1.85)$, for 67 preparations of cetuximab + $0.71 \%(\mathrm{DS}=1.13$ ) and for 152 preparations of trastuzumab $-0.57 \%$ $(\mathrm{DS}=1.8)$
In the period under review, $85.9 \mathrm{~g}$ of bevacizumab, $37.5 \mathrm{~g}$ of cetuximab and $43.8 \mathrm{~g}$ trastuzumab were prepared using material that would previously have been discarded. This provided considerable saving for the three drugs $(€ 29,893)$ which corresponds to approximately $€ 90,000$ per year.

Conclusions The centralised system and the use of APOTECAchemo is successful both in terms of patient and operator safety and cost benefit for the Hospital.

No conflict of interest.

\section{TCH-005 DEVELOPING A SAFE SYSTEM TO PRESCRIBE, PREPARE AND ADMINISTER CYTOSTATIC DRUGS}

doi:10.1136/ejhpharm-2013-000276.196

S Ruiz-Fuentes, S Belda-Rustarazo, C García-Fernandez, C Gómez-Peña, C MedardeCaballero, C Fernández-López, D Blanquez-Martínez, A Caballero-Romero. Hospital Universitario San Cecilio, Pharmacy, Granada, Spain

Background As the cytostatic medicines are a group of drugs with a narrow therapeutic index, it is necessary to develop new mechanisms to improve safety from prescription to administration in the hospital in order to avoid fatal errors.

Purpose To develop a system that ensures that the prescription process, production and administration of cytostatic drugs meet the criteria: right patient, medicine, dosage, route of administration and time.

Materials and Methods Along with the centralization of drugs preparation in the pharmacy service, a computer system has been designed for the management of the administration of cytostatic drugs consisting of: portable digital assistant (PDA) with barcode reader, label printer for barcoded medicines, patient-identifying wristband and dedicated software for verifying and recording administration.

Results Every chemotherapy prescription is sent to the cytotoxic admixture unit mixer where it is validated by a pharmacist checking the following items: name and number from the patient history, diagnosis, stage, line of treatment, drugs, dose and route of administration. The computer programme generates drug labels containing the bar code which identifies the preparation. Each patient has a label with the bar code of the history number. Before the administration of each cycle, the responsible nurse has to read the patient bar code with the PDA. The drug and the right order for that patient will appear on the screen of the device. Nurses should read the bar code of each drug to be administered and the system cheques that it is the right medicine and order, alerting visually and acoustically if error occurs. The system records the nurse and time of each drug administration.

Conclusions The project was implemented due to the need for safety mechanisms in the management of high-risk medicines, as cancer treatments are group of drugs with a narrow therapeutic index.

The system cheques the safety in five key areas: patient, medicine, dose, route of administration and time.

No conflict of interest.

\section{TCH-006 DEVELOPMENT AND VALIDATION OF 3 METHODS - UV SPECTROPHOTOMETRY, FLOW INJECTION ANALYSIS AND LIOUID CHROMATOGRAPHY - FOR THE CONTROL OF NYSTATIN CAPSULES.}

doi:10.1136/ejhpharm-2013-000276.197

M Herold, A Nicolas, I May. CHU - Hôpitaux de Brabois Adultes, Pharmacie, Vandoeuvre Cedex, France

Background As an alternative to amphotericin B used for selective digestive decontamination, physicians asked the Hospital Pharmacy for the preparation of nystatin capsules, 500,000 IU. 